\title{
An Update on the Components and Functions of Bone Marrow Niche
}

Ghazanfari R and Behravan J*

Biotechnology Research Center, Mashhad University of Medical Sciences, Iran

*Correspondling author: Behravan J , Biotechnology Research Center, Mashhad University of Medical

Sciences, Mashhad, Iran

Received: October 01, 2017; Accepted: October 25, 2017; Published: November 20, 2017

\section{Keywords}

Bone marrow; Niche; HSCs

\section{Editorial}

In the embryonic stage, hematopoiesis happens in different anatomical locations; including liver, spleen, yolk sac, aorta-gonadmesonephros, and bone marrow, while the main location for hematopoiesis during the adulthood is the bone marrow. In the bone marrow, a highly supportive microenvironment which is called niche maintains the best conditions for the existence of hematopoietic stem cells (HSCs).

This dynamic microenvironment provides all the cellular components and molecular signals that are essential for HSC maintenance and ensures the rapid blood production for daily needs $[1,2]$. Most of our new vision about niche comes from advanced imaging technologies, different transgenic mouse models and in vivo lineage tracing studies.

Through these, researchers are trying to discover the cellular and molecular components of niche. The major cellular constituents of the niche are mesenchymal stem cells (MSCs) and their derivatives including perivascular cells, osteoblasts and adipocytes, and also endothelial cells and Schwann cells.

The cellular constituents of niche produce several soluble factors including growth factors and cytokines providing regulatory signals required for hematopoiesis. These soluble factors include stem cell factor (SCF), transforming growth factor (TGF- $\beta$ ), C-X-C motif chemokine ligand 12 (CXCL12), angiopoietin-1, granulocyte-colony stimulating factor (G-CSF), Wnt ligands, and thrombopoietin (TPO) $[1,3]$. In the bone marrow microenvironment, two distinct niches have been proposed.

First, the endosteal niche which harbor a minority of HSCs and seemingly keeps the HSCs in a quiescent state, although this issue is still under debate. Second vascular niche that is mainly comprised of cells with MSC characteristics (Nestin ${ }^{+}$MSCs, CXCL12-abundant reticular (CAR) cells, Leptin receptor ${ }^{+}$cells) and prepare the predominant site for HSCs localization. Other vascular niche candidates are arterioles enclosed by pericytes and endothelial cells $[4,5]$.

In the homeostasis state, niche precisely controls the HSC fate decisions and adjusts a balance between the rate of HSC self-renewal and differentiation. Mobilization and homing of HSCs which are mirror events are important physiological processes and are highly controlled by niche elements [6]. The crucial role of niche is emphasized by the fact that deregulation of the niche function can lead to initiation and development of hematopoietic malignancies $[7,8]$. In spite of the great advancements in understanding the niche function, there are open questions that need to be answered.

Both MSCs and HSCs are heterogeneous populations. It is still not fully clear if different HSC subpopulations need different niches, what is the exact composition and localization of each niche and how much is the extent of overlap between different niches in the bone marrow.

Revealing the exact molecular mechanisms and signaling pathways that control bone marrow niche functionality has a particular importance in regenerative medicine in which production of blood cells in patients with deficient hematopoiesis is an ultimate goal.

\section{References}

1. Pleyer L, Valent P, Greil R. Mesenchymal Stem and Progenitor Cells in Normal and Dysplastic Hematopoiesis-Masters of Survival and Clonality? Int J Mol Sci. 2016; 17

2. Morrison SJ, Scadden DT. The bone marrow niche for haematopoietic stem cells. Nature. 2014; 505: 327-334.

3. Yu VW, Scadden DT. Hematopoietic Stem Cell and Its Bone Marrow Niche Curr Top Dev Biol. 2016; 118: 21-44

4. Beerman I, Luis TC, Singbrant S, Lo Celso C, Mendez-Ferrer S. The evolving view of the hematopoietic stem cell niche. Exp Hematol. 2017; 50: 22-26.

5. Asada N, Takeishi S, Frenette PS. Complexity of bone marrow hematopoietic stem cell niche. Int J Hematol. 2017; 106: 45-54.

6. Suarez-Alvarez B, Lopez-Vazquez A, Lopez-Larrea C. Mobilization and homing of hematopoietic stem cells. Adv Exp Med Biol. 2012; 741: 152-70.

7. Garcia-Garcia A, de Castillejo CL, Mendez-Ferrer S. BMSCs and hematopoiesis. Immunol Lett. 2015; 168:129-135.

8. Korn C, Mendez-Ferrer S. Myeloid malignancies and the microenvironment. Blood. 2017; 129: 811-22. 